\title{
LOWER BOUNDS FOR THE CONSTANTS IN THE BOHNENBLUST-HILLE INEQUALITY: THE CASE OF REAL SCALARS
}

\author{
D. DINIZ, G. A. MUÑOZ-FERNÁNDEZ *, D. PELLEGRINO, AND J. B. SEOANE-SEPÚLVEDA*
}

Abstract. The Bohnenblust-Hille inequality was obtained in 1931 and (in the case of real scalars) asserts that for every positive integer $N$ and every $m$-linear mapping $T: \ell_{\infty}^{N} \times \cdots \times \ell_{\infty}^{N} \rightarrow$ $\mathbb{R}$ one has

$$
\left(\sum_{i_{1}, \ldots, i_{m}=1}^{N}\left|T\left(e_{i_{1}}, \ldots, e_{i_{m}}\right)\right|^{\frac{2 m}{m+1}}\right)^{\frac{m+1}{2 m}} \leq C_{m}\|T\|,
$$

for some positive constant $C_{m}$. Since then, several authors obtained upper estimates for the values of $C_{m}$. However, the novelty presented in this short note is that we provide lower (and non-trivial) bounds for $C_{m}$.

\section{INTRODUCTION}

The Bohnenblust-Hille inequality (see 1), for real scalars asserts that for every positive integer $N$ and every $m$-linear mapping $T: \ell_{\infty}^{N} \times \cdots \times \ell_{\infty}^{N} \rightarrow \mathbb{R}$ there is a positive constant $C_{m}$ such that

$$
\left(\sum_{i_{1}, \ldots, i_{m}=1}^{N}\left|T\left(e_{i_{1}}, \ldots, e_{i_{m}}\right)\right|^{\frac{2 m}{m+1}}\right)^{\frac{m+1}{2 m}} \leq C_{m}\|T\| .
$$

The BohnenblustHille inequality has important applications in various fields of analysis. For details and references we mention [4. When $m=2$ it is interesting to note that the Bohnenblust-Hille inequality is precisely the well-known Littlewood's $4 / 3$ inequality 8 .

Since the 1930's many authors have obtained estimates for upper bounds of $C_{m}$ in the case of real and complex scalars (see, e.g., [1,2,4,5, 7, 9, 10]). The constants of the polynomial version of the Bohnenblust-Hille inequality (complex case) were recently investigated in 3 . Until now, the more accurate upper bounds for $C_{m}$ (real case) were given in [10]:

\begin{tabular}{c|c}
$m$ & upper bounds for $C_{m}$ \\
\hline 2 & $\sqrt{2} \approx 1.414$ \\
3 & $2^{\frac{20}{24}} \approx 1.782$ \\
4 & $2^{\frac{32}{32}}=2$ \\
5 & $2^{\frac{48}{40}} \approx 2.298$ \\
6 & $2^{\frac{64}{48}} \approx 2.520$ \\
7 & $2^{\frac{84}{56}} \approx 2.828$ \\
8 & $2^{\frac{104}{64}} \approx 3.084$ \\
9 & $2^{\frac{128}{72}} \approx 3.429$ \\
10 & $2^{\frac{152}{80}} \approx 3.732$
\end{tabular}

Also, it has very recently been proved ([6]) that the sequence of constants $\left(C_{m}\right)_{m}$ has the best possible asymptotic behavior, that is

$$
\lim _{m \rightarrow \infty} \frac{C_{m}}{C_{m-1}}=1
$$

2010 Mathematics Subject Classification. 46G25, 47H60.

Key words and phrases. Absolutely summing operators, Bohnenblust-Hille Theorem.

* The second and fourth authors were supported by the Spanish Ministry of Science and Innovation (grant MTM2009-07848). 
However, and to the best of our knowledge, there is absolutely no work presenting estimates for (non-trivial) lower bounds for the constants $C_{m}$. In this short note we obtain lower bounds for $C_{m}$ which we believe are (specially) interesting for the cases $m=2,3,4,5$.

In the following $e_{k}$ denotes the $k$ th canonical vector in $\mathbb{R}^{N}$.

2. THE CASE $m=2$

Let $T_{2}: \ell_{\infty}^{2} \times \ell_{\infty}^{2} \rightarrow \mathbb{R}$ be defined as

$$
T_{2}(x, y)=x_{1} y_{1}+x_{1} y_{2}+x_{2} y_{1}-x_{2} y_{2}
$$

Note that $\left\|T_{2}\right\|=2$. In fact,

$$
\begin{aligned}
\left|T_{2}(x, y)\right| & =\left|x_{1}\left(y_{1}+y_{2}\right)+x_{2}\left(y_{1}-y_{2}\right)\right| \\
& \leq\|x\|\left(\left|y_{1}+y_{2}\right|+\left|y_{1}-y_{2}\right|\right) \\
& =2\|x\| \max \left\{\left|y_{1}\right|,\left|y_{2}\right|\right\} \\
& =2\|x\|\|y\|
\end{aligned}
$$

and since $T_{2}\left(e_{1}+e_{2}, e_{1}+e_{2}\right)=2$ it follows that $\left\|T_{2}\right\|=2$.

Now the inequality

can be re-written as

$$
\left(\sum_{i_{1}, i_{2}=1}^{2}\left|T_{2}\left(e_{i_{1}}, e_{i_{2}}\right)\right|^{\frac{4}{3}}\right)^{\frac{3}{4}} \leq C_{2}\left\|T_{2}\right\|
$$

$$
4^{3 / 4} \leq 2 C_{2}
$$

which gives

$$
C_{2} \geq 2^{1 / 2} .
$$

Since it is well-known that $C_{2} \leq 2^{1 / 2}$, we conclude that $C_{2}=2^{1 / 2}$, but this result seems to be already known.

\section{THE CASE $m=3$}

Now, let $T_{3}: \ell_{\infty}^{4} \times \ell_{\infty}^{4} \times \ell_{\infty}^{4} \rightarrow \mathbb{R}$ given by

$$
T_{3}(x, y, z)=\left(z_{1}+z_{2}\right)\left(x_{1} y_{1}+x_{1} y_{2}+x_{2} y_{1}-x_{2} y_{2}\right)+\left(z_{1}-z_{2}\right)\left(x_{3} y_{3}+x_{3} y_{4}+x_{4} y_{3}-x_{4} y_{4}\right) \text {. }
$$

We have

$$
\begin{aligned}
\left|T_{3}(x, y, z)\right| & =\left|\left(z_{1}+z_{2}\right)\left(x_{1} y_{1}+x_{1} y_{2}+x_{2} y_{1}-x_{2} y_{2}\right)+\left(z_{1}-z_{2}\right)\left(x_{3} y_{3}+x_{3} y_{4}+x_{4} y_{3}-x_{4} y_{4}\right)\right| \\
& \leq\left|z_{1}+z_{2}\right|\left(\left|x_{1}\right|\left|y_{1}+y_{2}\right|+\left|x_{2}\right|\left|y_{1}-y_{2}\right|\right)+\left|z_{1}-z_{2}\right|\left(\left|x_{3}\right|\left|y_{3}+y_{4}\right|+\left|x_{4}\right|\left|y_{3}-y_{4}\right|\right) \\
& \leq\|x\|\left\{\left|z_{1}+z_{2}\right|\left(\left|y_{1}+y_{2}\right|+\left|y_{1}-y_{2}\right|\right)+\left|z_{1}-z_{2}\right|\left(\left|y_{3}+y_{4}\right|+\left|y_{3}-y_{4}\right|\right)\right\} \\
& =2\|x\|\left\{\left|z_{1}+z_{2}\right| \max \left\{\left|y_{1}\right|,\left|y_{2}\right|\right\}+\left|z_{1}-z_{2}\right| \mid \max \left\{\left|y_{3}\right|,\left|y_{4}\right|\right\}\right\} \\
& \leq 2\|x\|\|y\|\left(\left|z_{1}+z_{2}\right|+\left|z_{1}-z_{2}\right|\right) \\
& =4\|x\|\|y\| \max \left\{\left|z_{1}\right|,\left|z_{2}\right|\right\} \\
& \leq 4\|x\|\|y\|\|z\| .
\end{aligned}
$$

Since $T_{3}\left(e_{1}+e_{2}+e_{3}, e_{1}+e_{2}+e_{3}, e_{1}+e_{2}+e_{3}\right)=4$, then $\left\|T_{3}\right\|=4$. Also

$$
\left(\sum_{i_{1}, i_{2}, i_{3}=1}^{4}\left|T_{3}\left(e_{i_{1}}, e_{i_{2}}, e_{i_{3}}\right)\right|^{\frac{6}{4}}\right)^{\frac{4}{6}} \leq C_{3}\left\|T_{3}\right\|
$$

becomes

which gives

$$
16^{2 / 3} \leq 4 C_{3}
$$

$$
C_{3} \geq 2^{2 / 3} \approx 1.587 .
$$


4. THE CASE $m=4$

In this case, let us consider $T_{4}: \ell_{\infty}^{8} \times \ell_{\infty}^{8} \times \ell_{\infty}^{8} \times \ell_{\infty}^{8} \rightarrow \mathbb{R}$ given by

$$
\begin{aligned}
& T_{4}(x, y, z, w)= \\
& =\left(w_{1}+w_{2}\right)\left(\begin{array}{c}
\left(z_{1}+z_{2}\right)\left(x_{1} y_{1}+x_{1} y_{2}+x_{2} y_{1}-x_{2} y_{2}\right) \\
+\left(z_{1}-z_{2}\right)\left(x_{3} y_{3}+x_{3} y_{4}+x_{4} y_{3}-x_{4} y_{4}\right)
\end{array}\right) \\
& +\left(w_{1}-w_{2}\right)\left(\begin{array}{c}
\left(z_{3}+z_{4}\right)\left(x_{5} y_{5}+x_{5} y_{6}+x_{6} y_{5}-x_{6} y_{6}\right) \\
+\left(z_{3}-z_{4}\right)\left(x_{7} y_{7}+x_{7} y_{8}+x_{8} y_{7}-x_{8} y_{8}\right) .
\end{array}\right)
\end{aligned}
$$

As in Sections 2 and 3 we see that $\left\|T_{4}\right\|=8$ and from (1.1) we obtain

$$
64^{5 / 8} \leq 8 C_{4} \text {. }
$$

Hence

$$
C_{4} \geq 2^{3 / 4} \approx 1.681
$$

\section{The general Case}

From the previous results it is not difficult to prove that in general

$$
C_{m} \geq 2^{\frac{m-1}{m}}
$$

for every $m \geq 2$. Indeed, let us define the $m$-linear forms $T_{m}: \ell_{\infty}^{2^{m-1}} \times \stackrel{m}{.} \times \ell_{\infty}^{2^{m-1}} \rightarrow \mathbb{R}$ by induction as

$$
\begin{aligned}
T_{2}\left(x_{1}, x_{2}\right)= & x_{1}^{1} x_{2}^{1}+x_{1}^{1} x_{2}^{2}+x_{1}^{2} x_{2}^{1}-x_{1}^{2} x_{2}^{2} \\
T_{m}\left(x_{1}, \ldots, x_{m}\right)= & \left(x_{m}^{1}+x_{m}^{2}\right) T_{m-1}\left(x_{1}, \ldots, x_{m-1}\right) \\
& +\left(x_{m}^{1}-x_{m}^{2}\right) T_{m-1}\left(B^{2^{m-2}}\left(x_{1}\right), B^{2^{m-2}}\left(x_{2}\right), B^{2^{m-3}}\left(x_{3}\right) \ldots, B^{2}\left(x_{m-1}\right)\right),
\end{aligned}
$$

where $x_{k}=\left(x_{k}^{n}\right)_{n} \in \ell_{\infty}^{2^{m-1}}$ for $1 \leq k \leq m, 1 \leq n \leq 2^{m-1}$ and $B$ is the backward shift operator in $\ell_{\infty}^{2^{m-1}}$. It has been proved in Section 2 that $\left\|T_{2}\right\|=2$. If we assume that $\left\|T_{m-1}\right\|=2^{m-2}$, then

$$
\begin{aligned}
\mid T_{m}\left(x_{1}, \ldots, x_{m}\right) \leq & \left|x_{m}^{1}+x_{m}^{2}\right|\left|T_{m-1}\left(x_{1}, \ldots, x_{m-1}\right)\right| \\
& +\left|x_{m}^{1}-x_{m}^{2} \| T_{m-1}\left(B^{2^{m-2}}\left(x_{1}\right), B^{2^{m-2}}\left(x_{2}\right), B^{2^{m-3}}\left(x_{3}\right) \ldots, B^{2}\left(x_{m-1}\right)\right)\right| \\
\leq & 2^{m-2}\left[\left|x_{m}^{1}+x_{m}^{2}\right|\left\|x_{1}\right\| \cdots\left\|x_{m-1}\right\|\right. \\
& \left.+\left|x_{m}^{1}-x_{m}^{2}\right|\left\|B^{2^{m-2}}\left(x_{1}\right)\right\|\left\|B^{2^{m-2}}\left(x_{2}\right)\right\|\left\|B^{2^{m-1}}\left(x_{3}\right)\right\| \cdots\left\|B^{2}\left(x_{m-1}\right)\right\|\right] \\
\leq & 2^{m-2}\left[\left|x_{m}^{1}+x_{m}^{2}\right|+\left|x_{m}^{1}-x_{m}^{2}\right|\right]\left\|x_{1}\right\| \cdots\left\|x_{m-1}\right\| \\
= & 2^{m-1}\left\|x_{1}\right\| \cdots\left\|x_{m-1}\right\| \max \left\{\left|x_{m}^{1}\right|,\left|x_{m}^{2}\right|\right\} \\
\leq & 2^{m-1}\left\|x_{1}\right\| \cdots\left\|x_{m}\right\| .
\end{aligned}
$$

This induction argument shows that $\left\|T_{m}\right\| \leq 2^{m-1}$ for all $m \in \mathbb{N}$. Using a similar induction argument it is easy to prove that $T_{m}\left(x_{1}, \ldots, x_{m}\right)=2^{m-1}$ for $x_{1}, \ldots, x_{m}$ such that $\left\|x_{1}\right\|=\ldots=$ $\left\|x_{m}\right\|=1$ and $x_{j}^{k}=1$ with $1 \leq j, k \leq m$, which proves that $\left\|T_{m}\right\|=2^{m-1}$ for all $m \in \mathbb{N}$.

On the other hand from (1.1) we have

$$
\left(\sum_{i_{1}, \ldots, i_{m}=1}^{2^{m-1}}\left|T_{m}\left(e_{i_{1}}, \ldots, e_{i_{m}}\right)\right|^{\frac{2 m}{m+1}}\right)^{\frac{m+1}{2 m}} \leq C_{m}\left\|T_{m}\right\|=2^{m-1} C_{m} .
$$

To finish we shall prove that $\left|T_{m}\left(e_{i_{1}}, \ldots, e_{i_{m}}\right)\right|$ is either 0 or 1 and that $\left|T_{m}\left(e_{i_{1}}, \ldots, e_{i_{m}}\right)\right|=1$ for exactly $4^{m-1}$ choices of the vectors $e_{i_{1}}, \ldots, e_{i_{m}}$. Working again by induction, the reader can easily check that the latter is true for $m=2$ (see Section 2). If we assume that the result is true for $m-1$ and $e_{i_{1}}, \ldots, e_{i_{m-1}}$ is one of the $4^{m-2}$ choices of the unit vectors such that $\left|T_{m-1}\left(e_{i_{1}}, \ldots, e_{i_{m-1}}\right)\right|=1$, then using the definition of $T_{m}$

$$
\begin{aligned}
\left|T_{m}\left(e_{i_{1}}, \ldots, e_{i_{m-1}}, e_{k}\right)\right| & =\left|T_{m-1}\left(e_{i_{1}}, \ldots, e_{i_{m-1}}\right)\right|=1, \\
\left|T_{m}\left(e_{i_{1}+2^{m-2}}, e_{i_{2}+2^{m-2}}, e_{i_{3}+2^{m-3}} \ldots, e_{i_{m-1}+2}, e_{k}\right)\right| & =\left|T_{m-1}\left(e_{i_{1}}, \ldots, e_{i_{m-1}}\right)\right|=1,
\end{aligned}
$$


for $k=1,2$. Hence we have found $4 \cdot 4^{m-2}=4^{m-1}$ choices of unit vector at which $\left|T_{m}\right|$ takes the value 1 . A simple inspection of the problem shows that $\left|T_{m}\right|$ vanishes at any other choice of canonical vectors.

\section{FinAl REMARKS}

Notice that our estimate $2^{\frac{m-1}{m}}$ seems inaccurate as $m \rightarrow \infty$ since it is a common feeling that the optimal values for the constants $C_{m}$ should tend to infinity as $m \rightarrow \infty$. However, and as a matter of fact, we must say that this common feeling seems to be just supported by the estimates of the upper bounds for $C_{m}$ obtained throughout the last decades, but there seems to be not any particular result supporting this "fact". In any case (and at least for $m=2,3,4,5$ ) our estimates are clearly interesting. Summarizing:

$$
\begin{aligned}
& C_{2}=\sqrt{2} \\
1.587 \leq C_{3} & \leq 1.782 \\
1.681 \leq C_{4} & \leq 2 \\
1.741 \leq C_{5} & \leq 2.298 .
\end{aligned}
$$

We also conclude that $C_{3}>C_{2}$, which seems to be not known until now.

\section{REFERENCES}

[1] H. F. Bohnenblust and E. Hille, On the absolute convergence of Dirichlet series, Ann. of Math. (2) 32 (1931), no. 3, 600-622.

[2] A. M. Davie, Quotient algebras of uniform algebras, J. London Math. Soc. (2) 7 (1973), 31-40.

[3] A. Defant, L. Frerick, J. Ortega-Cerdà, M. Ounaïes, and K. Seip, The Bohnenblust-Hille inequality for homogeneous polynomials is hypercontractive, Ann. of Math. (2) 174 (2011), 485-497.

[4] A. Defant, D. Popa, and U. Schwarting, Coordinatewise multiple summing operators in Banach spaces, J. Funct. Anal. 259 (2010), no. 1, 220-242.

[5] A. Defant and P. Sevilla-Peris, A new multilinear insight on Littlewood's 4/3-inequality, J. Funct. Anal. 256 (2009), no. 5, 1642-1664.

[6] D. Diniz, G. A. Muñoz-Fernández, D. Pellegrino, and J. B. Seoane-Sepúlveda, The asymptotic growth of the constants in the Bohnenblust-Hille inequality is optimal, arXiv:1108.1550v2 [math.FA].

[7] S. Kaijser, Some results in the metric theory of tensor products, Studia Math. 63 (1978), no. 2, 157-170.

[8] J. E. Littlewood, On bounded bilinear forms in an infinite number of variables, Q. J. Math. 1 (1930), 164-174.

[9] G. A. Muñoz-Fernández, D. Pellegrino, and J. B. Seoane-Sepúlveda, Estimates for the asymptotic behavior of the constants in the Bohnenblust-Hille inequality, Linear Multilinear A., In Press.

[10] D. Pellegrino and J. B. Seoane-Sepúlveda, New upper bounds for the constants in the Bohnenblust-Hille inequality, J. Math. Anal. Appl. 386 (2012), 300-307.

Unidade Academica de Matemática e Estatística,

Universidade Federal de Campina Grande,

Caixa Postal 10044,

Campina Grande, 58429-970, Brazil.

E-mail address: diogo@dme.ufcg.edu.br

Departamento de Análisis Matemático,

Facultad de Ciencias Matemáticas,

Plaza de Ciencias 3,

Universidad COMPlutense de Madrid,

MADRID, 28040, SPAIN.

E-mail address: gustavo_fernandez@mat.ucm.es

Departamento de Matemática,

Universidade Federal da Paraíba,

58.051-900 - JoÃo PESSOA, BrazIL.

E-mail address: pellegrino@pq.cnpq.br and dmpellegrino@gmail.com

Departamento de Análisis Matemático,

Facultad de Ciencias Matemáticas,

Plaza de Ciencias 3,

Universidad Complutense de Madrid,

MADRID, 28040, SPAIN.

E-mail address: jseoane@mat.ucm.es 